\title{
Desarrollo de la capacidad de innovación en procesos de servicio al cliente del departamento de matrícula en instituciones de educación superior en Barranquilla, Colombia
}

\author{
Yolanda Vega-Sampayo, Enohemit Olivero-Vega y Emiro Gastelbondo-Gómez \\ Facultad de Administración y Negocios, Universidad Simón Bolívar, Barranquilla-Colombia. \\ (correo-e: yvega11@unisimonbolivar.edu.co, eolivero@unisimonbolivar.edu.co).
}

Recibido Abr. 7, 2020; Aceptado Jun. 3, 2020; Versión final Jul. 20, 2020, Publicado Oct. 2020

\begin{abstract}
Resumen
Este estudio tuvo como finalidad analizar la capacidad de innovación en procesos de servicio al cliente del departamento de matrícula en las instituciones de educación superior en Barranquilla (Colombia). Esta investigación fue de enfoque cuantitativo, de alcance correlacional, mediante una encuesta estructurada por treinta ítems en escala tipo Likert, aplicada a 398 estudiantes para evaluar la relación entre capacidad de innovación y servicio al cliente. El análisis estadístico mostró asociaciones relevantes en los factores evaluados de capacidad de innovación con los factores del servicio al cliente. Sin embargo, las relaciones más significativas se presentaron en los factores de explotación y ambidestreza, presentándose en menor grado la explotación. Se concluye que la capacidad de innovación en las instituciones se desarrolla en gran medida, sin embargo, es importante seguir trabajando, específicamente en la explotación para ofrecer un servicio excepcional que permita satisfacer plenamente las necesidades del cliente.
\end{abstract}

Palabras clave: capacidad de innovación; factores organizacionales; servicio al cliente; instituciones de educación superior

\section{Innovation capacity development in customer service processes of enrollment departments in higher education institutions from Barranquilla, Colombia}

\begin{abstract}
The purpose of this study was to analyze the capacity of innovation in customer service processes of enrollment departments in higher education institutions from Barranquilla (Colombia). This research was of quantitative approach and correlational in scope. A survey structured with thirty items on a Likert-type scale was applied to 398 students to assess the relationship between innovation capacity and customer service. The statistical analysis showed relevant associations between innovation capacity factors and customer service factors. However, the most significant relationships were found in the exploitation and ambidexterity factors, with exploitation being presented to a lesser degree. It is concluded that the capacity for innovation in the institutions sampled is developed to a great extent. However, it is important to continue working on offering an exceptional service that fully satisfies client needs.
\end{abstract}




\section{INTRODUCCIÓN}

Actualmente las organizaciones viven inmersas en un entorno versátil que las obliga a transformarse para sostenerse en el mercado, ya que el medio competitivo en el que operan las induce a generar innovación, emplear tecnología y gestión de conocimiento como ejes claves del crecimiento y adaptación organizacional (Hosseini et al., 2019). Por ello Schumpeter (1942), plantea la innovación como factor decisivo del desarrollo económico, clasificándola en innovación de productos, nuevas formas de organizar la empresa, nuevos métodos de producción, nuevas fuentes de suministro y exploración de mercados (Jordán, 2011). A su vez, el Manual de Oslo (OCDE y Eurostat, 2006), la clasifican en innovación en productos, procesos empresariales y de marketing, para aumentar el nivel de cambios en las actividades de una organización; permitiendo innovar en distintos enfoques empresariales, fortaleciendo esas áreas que requieren mejoras en procesos científicos, técnicos, comerciales y financieros, necesarios para el desarrollo comercial exitoso de nuevos productos 0 servicios. Pero, Hayek (1945) dice que la innovación está al exterior organizacional y el orden económico racional se define mediante el conocimiento sobre los escenarios de los que se debe hacer uso, y se percibe de forma inexistente, ni se presenta de forma integrada. A esto, Schumpeter (1942) afirma que la innovación científica básica tiene un rol secundario frente a la innovación aplicada al interior de una organización, señala que el principal agente de innovación está al interior de esta y no en su exterior (Kohler y González, 2014).

Por su parte, Da Silva et al., (2016) resaltan que el trabajo entre distintas áreas empresariales con el mercado externo, se convierte en estímulos necesarios para conocer las necesidades del cliente, y poder generar innovaciones mediante un proceso sistemático, dirigido, ejecutado y supervisado por el líder gerencial para lograr un buen manejo empresarial (Espinosa et al., 2008). Ante esto, las Instituciones de Educación Superior llamadas (IES) en adelante no son ajenas a ello, como productoras del conocimiento se centran en conocer sus capacidades innovadoras para direccionar adecuadamente sus estrategias en la satisfacción eficaz del cliente; ya que el reto de las IES reside en la diferenciación de sus procesos organizacionales y es aquí donde un estudio de capacidad de innovación debe responder a esa necesidad; y las IES contribuyen al facilitar recursos necesarios a los innovadores al generar un cambio institucional, teniendo en cuenta los recursos que favorecen la innovación en aspectos que mejoren los procesos de las IES (Benneworth et al., 2017).

De esta forma, Michelino et al., (2015) indican que la capacidad receptiva, adaptativa con socios académicos y del sector externo son necesarios en el proceso innovador, para responder a problemas actuales y aumentar la capacidad innovadora de las IES (Reichert, 2019). Ya que, las experiencias negativas que experimenta el estudiante al realizar trámites de gestión académica, como (información, inscripciones, matrícula y derecho a grado, etc); se debe a eventos adversos que presenta el estudiante en dichos trámites, por esa razón, es preciso que su experiencia sea positiva para evitar consecuencias que afecten a las IES. Por tanto, el proceso de innovación en IES debe fortalecerse siempre y emplear estrategias para sostenerla en el mercado las obliga abordar retos de innovación incremental y disruptivos uniendo el enfoque institucional para ofrecer un servicio eficaz; adicionalmente, el proceso de éxito innovador se centra en factores asociados al entorno y a la capacidad organizacional para su desarrollo (Chen y Yuan, 2007; Mohamad et al., 2010). Esos factores que indican el grado de capacidad de innovación son innovación de productos, procesos, inversión en l+D y canales de distribución (Sozbilir, 2018); favoreciendo la generación y transformación de ideas para que la empresa se beneficie de las oportunidades del mercado (Yam, 2004; Arias et al., 2015).

Además, la capacidad de innovación en la organización cultiva, asimila, adapta y transforma la tecnología en hábitos gerenciales, operacionales y transaccionales de forma eficaz para que sea valorada como innovadora, logrando los beneficios esperados con el desarrollo de habilidades que permiten un desempeño competitivo en función de una buena gestión organizacional (García et al., 2014). A su vez, Acosta y Fischer (2013), plantean una clasificación del proceso de capacidad de innovación en función del progresivo conocimiento, definidos como explotación, exploración y ambidestreza que permiten aprovechar recursos y capacidades organizacionales para ofrecer productos o servicios innovadores. Asimismo, se analizan tres factores de capacidad de innovación para organizaciones de servicio como capacidad de innovación de orientación al cliente (CIOC), permite la destreza de responder a problemas del cliente de forma innovadora; capacidad de orientación al mercadeo (CIOM), es la habilidad de implementar programas de mercadeo para promocionar servicios de forma innovadora y capacidad de innovación orientada a la tecnología, destreza de adoptar, crear e innovar con nuevos softwares, sistemas integrados y tecnologías actualizadas (García et al., 2014).

Respecto a la calidad del servicio al cliente se resalta el valor que tiene en una organización, ya que gestionar el servicio implica una satisfacción mutua que supere expectativas empresariales y del cliente. Para eso la organización debe interactuar y retroalimentar con el personal en todas las fases del proceso del servicio, para lograr resultados significativos (Gruca y Rego, 2005; Duque, 2005). Por eso, un servicio extraordinario no debe presentarse como ilusión organizacional, necesita generar significancia del servicio con factores que permitan entender las expectativas del cliente, como fiabilidad al ofrecer un servicio eficaz, infraestructura de contar con buenos equipos e instalaciones físicas, capacidad de respuesta e Interacción humana al atender 
y escuchar al cliente (Berry, 2013). Frente a esto, las IES desarrollan sus actividades en un entorno complejo, acentuado en la oferta de estudios a nivel nacional e internacional, por ello la alta gerencia debe adaptarse a las exigencias del entorno, ajustando el servicio en función a las necesidades del cliente para fidelizarlo con una oferta de servicios de alto valor agregado, que mantenga la competitividad la cual se centra cada vez más en la atención y preferencia del cliente (Botía y Orozco, 2012). Además, Narver et al., (2004) dicen que un cliente satisfecho influye positivamente en el medio económico y competitivo organizacional frente al mercado, gracias a una excelente experiencia se logra alta demanda de servicios y a un bajo costo publicitario.

\section{METODOLOGÍA}

Las variables estudiadas fueron servicio al cliente y capacidad de innovación para conocer su influencia en procesos de servicio al cliente del departamento de matrículas de las IES en Barranquilla Colombia. Con la aplicación del enfoque cuantitativo mediante técnicas de medición numérica y el análisis estadístico en el desarrollo del estudio general de la investigación, para analizar patrones de comportamiento y comprobar la teoría abordada (Hernández et al., 2014). De alcance correlacional orientada a conocer la relación entre las variables estudiadas, la población fueron 65310 estudiantes pertenecientes a las IES de carácter privado de Barranquilla, registradas legalmente por el Sistema Nacional de Información de la Educación Superior (SNIES) hasta abril del año 2018. El muestreo fue aleatorio estratificado en varios programas académicos (Contaduría Pública, Derecho, Administración de Empresas, Comercio y Negocios Internacionales, Mercadeo y Publicidad, Comunicación Social, Ingenierías de sistemas, Industrial, Mecánica y Civil, Medicina, Fonoaudiología, Odontología, Radiología, Fisioterapia, Trabajo Social y Psicología), de las once IES seleccionadas de acuerdo a lo establecido por el Ministerio de Educación Nacional de Colombia, quien las reconoce como entidades oficialmente prestadoras del servicio de educación superior en el país. En este caso la muestra se determinó a través de la fórmula de Slovin, utilizada para calcular el tamaño de la muestra apropiada de una determinada población (Valbuena, 2017), ver fórmula 1.

$$
\mathrm{n}=\mathrm{N} /(1+\mathrm{Ne})^{2}=65310 /(1+00,5)^{2}=(65310)=398
$$

Para la estimación de la muestra se estableció un subgrupo de 398 estudiantes a través del muestreo aleatorio estratificado en distintos programas mencionados anteriormente representativo a la población estudiada. En donde $\mathrm{N}$ representó la población total investigada, e el error muestral y $\mathrm{n}$ el tamaño muestral a encuestar. Así, el peso de cada estrato lo determinó cada IES, y se estableció para el caso de la institución 1 de la siguiente manera ver la fórmula 2.

$$
\mathrm{Wi}=\mathrm{Ni} /(100 \%)=2501 / 65310(100 \%)=4 \%
$$

Posteriormente, para el resto de instituciones, se estableció la tabla 1 con la cual se obtuvo un total de 398 estudiantes encuestados

Tabla 1. Determinación de la muestra

\begin{tabular}{|l|l|l|l|}
\hline Instituciones (i) & Matriculados 2017 $\left(\mathrm{N}_{\mathrm{i}}\right)$ & Peso $\left(\mathrm{W}_{\mathrm{i}}\right)$ & Número de encuestas $\left(\mathrm{n}_{\mathrm{i}}\right)$ \\
\hline 1 & 2501 & $4 \%$ & 15 \\
\hline 2 & 10553 & $16 \%$ & 64 \\
\hline 3 & 11813 & $18 \%$ & 72 \\
\hline 4 & 611 & $1 \%$ & 4 \\
\hline 5 & 602 & $1 \%$ & 4 \\
\hline 6 & 4046 & $6 \%$ & 25 \\
\hline 7 & 854 & $1 \%$ & 5 \\
\hline 8 & 12974 & $20 \%$ & 79 \\
\hline 9 & 3332 & $5 \%$ & 20 \\
\hline 10 & 4539 & $7 \%$ & 28 \\
\hline 11 & 13485 & $21 \%$ & 82 \\
\hline Totales & $65310(\mathrm{~N})$ & $100 \%$ & 398 \\
\hline
\end{tabular}

La técnica usada para recoger los datos fue la encuesta conformada por un cuestionario estructurado por 30 ítems medidos en escala Likert, la evaluación se determinó así: del 1 al 5 (1 Totalmente en Desacuerdo; 2 en Desacuerdo; 3 ni de acuerdo, ni en desacuerdo, 4 De acuerdo y 5 Totalmente De acuerdo). A fin de evaluar y conocer la relación existente entre los factores de las variables estudiadas. Además, se contempló una metodología de análisis en tres fases de estadísticas: fase uno análisis preliminar de los datos obtenidos, para comprobar la fiabilidad del cuestionario y la coherencia de los ítems con las instrucciones y respuestas del encuestado, el método de fiabilidad fue el coeficiente alfa de Cronbach y Omega de Mcdonald. También, implicó el registro de los datos en el sistema SPSS versión 24 para verificar que no existirán errores aleatorios en el mismo. En la fase dos un análisis factorial exploratorio (AFE), usado para el desarrollo, validación y 
exploración del conjunto de variables comunes que explican las respuestas del cuestionario, definiendo el grado de libertad que refleja el dominio específico de contenido respecto a lo que se midió, con de validez (de contenido, de criterio y de constructo); la técnica utilizada en la extracción de los factores fue con estimaciones por componentes principales y rotación Varimax, para poder estudiar mejor la relación de las variables observadas. Finalmente, la fase tres un análisis factorial confirmatorio (AFC), para definir claramente los factores que se relacionan entre sí y los ítems que estaban relacionados con cada factor (Lloret et al., 2014).

\section{RESULTADOS}

El análisis de los resultados obtenidos muestra el nivel de relación entre las variables capacidad de innovación y servicio al cliente, en el departamento de matrícula de las IES en Barranquilla. En este caso, la capacidad de innovación se midió en función de 3 factores como exploración, explotación y ambidestreza organizacional. Para ello, se tomó como referencia las frecuencias relativas con la cual se logró analizar en menor grado la explotación de acuerdo a las respuestas de los estudiantes de las IES. Dentro de esos hallazgos se resalta el ítem donde la institución presenta constantes cambios en su departamento se observa un $48,4 \%$, aunque no es un porcentaje desfavorable, indica que la IES deben promover mejoras en sus procesos y recursos para obtener mejores resultados en los cambios. No obstante, la exploración presenta un $41,4 \%$ respecto a si la Institución ofrece servicios novedosos que superen las expectativas del cliente; por último, la ambidestreza evidencia un $45 \%$ de eficiencia en los procesos del servicio. Indicando la importancia de aprovechar recursos y capacidades en las IES para ofrecer servicios novedosos y beneficiarse de las oportunidades del mercado. De forma general es clave que las IES sigan apoyando desarrollo de capacidades organizacionales y el uso de sus recursos para brindar un servicio excepcional y responder a las exigencias del mercado. Ver tabla 2.

Tabla 2 Medición de factores de capacidad de innovación

\begin{tabular}{|c|c|c|c|c|c|c|}
\hline Factor & Ítems & 1 & 2 & 3 & 4 & 5 \\
\hline \multirow[t]{3}{*}{ Explotación } & $\begin{array}{l}\text { La Institución presenta diseños innovadores en el } \\
\text { manejo tecnológico para dar respuesta oportuna a las } \\
\text { solicitudes y tramites en el área de matrículas. }\end{array}$ & $6,3 \%$ & $10,9 \%$ & $19,9 \%$ & $48,4 \%$ & $14,5 \%$ \\
\hline & $\begin{array}{l}\text { El departamento de matrícula realiza acciones de } \\
\text { mejora en sus servicios para satisfacer las } \\
\text { necesidades del usuario. }\end{array}$ & $4,8 \%$ & $13,1 \%$ & $31,7 \%$ & $39,5 \%$ & $10,9 \%$ \\
\hline & $\begin{array}{l}\text { La Institución presenta constantes cambios en su } \\
\text { departamento de matrículas para ofrecer un servicio } \\
\text { excepcional a sus clientes. }\end{array}$ & $5,8 \%$ & $16,5 \%$ & $34,4 \%$ & $35,6 \%$ & $7,7 \%$ \\
\hline \multirow[t]{3}{*}{ Exploración } & $\begin{array}{l}\text { La Institución identifica los cambios del entorno para } \\
\text { adaptarse a las exigencias que este demanda. }\end{array}$ & $4,6 \%$ & $9,9 \%$ & $33,9 \%$ & $38,7 \%$ & $12,8 \%$ \\
\hline & $\begin{array}{l}\text { El departamento de matrículas de la Institución } \\
\text { emplea estrategias como la actualización constante } \\
\text { de su página para dar a conocer los servicios que } \\
\text { ofrece y de esta forma aprovechar las oportunidades } \\
\text { que se presentan en el mercado. }\end{array}$ & $3,9 \%$ & $6,8 \%$ & $21,3 \%$ & $45,3 \%$ & $22,8 \%$ \\
\hline & $\begin{array}{l}\text { La Institución asume riesgos en la implementación de } \\
\text { servicios novedosos que superen las expectativas del } \\
\text { cliente y les permita generar ventajas en el mercado. }\end{array}$ & $4,4 \%$ & $11,9 \%$ & $34,9 \%$ & $41,4 \%$ & $7,5 \%$ \\
\hline \multirow[t]{3}{*}{ Ambidestreza } & $\begin{array}{l}\text { Los cambios que implementa la Institución son } \\
\text { idóneos para ofrecer un mejor servicio que ofrece a } \\
\text { sus usuarios. }\end{array}$ & $4,6 \%$ & $8,0 \%$ & $30,8 \%$ & $42,6 \%$ & $14,0 \%$ \\
\hline & $\begin{array}{l}\text { La Institución está atenta a errores que puedan } \\
\text { suceder en su servicio ofrecido, para corregirlos y } \\
\text { resolver las dificultades con los usuarios. }\end{array}$ & $4,1 \%$ & $11,6 \%$ & $31,2 \%$ & $42,6 \%$ & $10,4 \%$ \\
\hline & $\begin{array}{l}\text { Las mejoras que lleva a cabo la Institución, en su } \\
\text { departamento de matrículas, permiten la eficiencia en } \\
\text { los procesos de servicio al cliente. }\end{array}$ & $4,6 \%$ & $12,3 \%$ & $28,3 \%$ & $45,8 \%$ & $9,0 \%$ \\
\hline
\end{tabular}

En el caso de los factores de servicio al cliente se presenta la fiabilidad en un $35,8 \%$, a su vez, la capacidad de respuesta en $39 \%$ y la interacción humana presentó puntos más críticos en un $32 \%$ que señalan la necesidad de ofrecer un servicio eficaz que responda a las solicitudes del estudiante, tomando en cuenta sus opiniones para realizar mejoras en el proceso de servicio, desarrollando innovaciones que direccionen a las IES hacia el éxito y lograr superar sus expectativas. También se observó la capacidad de orientación al cliente en $40 \%$, orientación al mercadeo con $45 \%$ y orientación a la tecnología en $36 \%$. Esto evidencia la necesidad de responder oportunamente a las necesidades del estudiante, mediante un buen manejo de las herramientas tecnológicas para promocionar los servicios ofrecidos en esa área y poder aumentar su nivel de satisfacción. 
Tabla 3 Medición de factores organizacionales de servicio al cliente

\begin{tabular}{|c|c|c|c|c|c|c|}
\hline Factor & Ítems & 1 & 2 & 3 & 4 & 5 \\
\hline \multirow[t]{2}{*}{$\begin{array}{l}\text { Orientación al } \\
\text { cliente }\end{array}$} & $\begin{array}{l}\text { Los servicios ofrecidos por la Institución dan muestra } \\
\text { de soluciones claras y oportunas para los clientes. }\end{array}$ & $4,6 \%$ & $11,4 \%$ & $32,0 \%$ & $40,7 \%$ & $11,4 \%$ \\
\hline & $\begin{array}{l}\text { La Institución explora las nuevas tendencias en el } \\
\text { mercado para presentar un mejor servicio a sus } \\
\text { usuarios. }\end{array}$ & $3,4 \%$ & $9,7 \%$ & $30,5 \%$ & $42,4 \%$ & $14,0 \%$ \\
\hline \multirow[t]{2}{*}{$\begin{array}{l}\text { Orientación al } \\
\text { mercadeo }\end{array}$} & $\begin{array}{l}\text { La institución ofrece publicidad continua en línea, para } \\
\text { dar a conocer sus servicios y despertar el interés del } \\
\text { cliente. }\end{array}$ & $3,1 \%$ & $6,8 \%$ & $16,9 \%$ & $46,5 \%$ & $26,6 \%$ \\
\hline & $\begin{array}{l}\text { Las herramientas publicitarias presentan claridad en } \\
\text { el servicio que ofrece la Institución. }\end{array}$ & $4,4 \%$ & $8,5 \%$ & $21,1 \%$ & $45,3 \%$ & $20,8 \%$ \\
\hline \multirow[t]{5}{*}{$\begin{array}{l}\text { Orientación a } \\
\text { la tecnología }\end{array}$} & $\begin{array}{l}\text { Los usuarios que ingresan en la plataforma de la } \\
\text { Institución son orientados de manera oportuna para } \\
\text { acceder a los servicios ofrecidos por la misma. }\end{array}$ & $4,4 \%$ & $13,6 \%$ & $22,8 \%$ & $41,4 \%$ & $17,9 \%$ \\
\hline & $\begin{array}{l}\text { La plataforma tecnológica implementada por el } \\
\text { departamento de matrículas hace más efectiva las } \\
\text { gestiones directas en los procesos de inscripción, } \\
\text { matrícula y legalización de documentos. }\end{array}$ & $3,9 \%$ & $12,6 \%$ & $20,1 \%$ & $46,0 \%$ & $17,4 \%$ \\
\hline & $\begin{array}{l}\text { Los recursos tecnológicos que ofrece la Institución } \\
\text { facilitan la gestión de los procesos académicos en } \\
\text { línea. }\end{array}$ & $3,4 \%$ & $10,9 \%$ & $21,1 \%$ & $45,3 \%$ & $19,4 \%$ \\
\hline & $\begin{array}{l}\text { La Institución utiliza tecnologías actualizadas para } \\
\text { mejorar sus procesos operacionales en el } \\
\text { departamento de matrículas. }\end{array}$ & $3,9 \%$ & $11,6 \%$ & $24,5 \%$ & $44,3 \%$ & $15,7 \%$ \\
\hline & $\begin{array}{l}\text { Las actualizaciones que la Institución realiza en la } \\
\text { plataforma son socializadas con los estudiantes para } \\
\text { próximos ingresos al sistema. }\end{array}$ & $7,5 \%$ & $17,2 \%$ & $28,8 \%$ & $36,1 \%$ & $10,4 \%$ \\
\hline \multirow[t]{3}{*}{ Fiabilidad } & $\begin{array}{l}\text { El personal del departamento de matrículas ofrece } \\
\text { atención eficiente para resolver los inconvenientes de } \\
\text { los usuarios de manera efectiva. }\end{array}$ & $7,3 \%$ & $14,5 \%$ & $26,6 \%$ & $38,3 \%$ & $13,3 \%$ \\
\hline & $\begin{array}{l}\text { La información que entrega el personal del } \\
\text { departamento de matrículas deja claridad en las } \\
\text { inquietudes que presentan los usuarios. }\end{array}$ & $5,8 \%$ & $16,0 \%$ & $30,8 \%$ & $35,8 \%$ & $11,6 \%$ \\
\hline & $\begin{array}{l}\text { Como usuario se siente seguro de la información que } \\
\text { suministra a la Institución, de que esta no será } \\
\text { utilizada indebidamente sino exclusivamente para } \\
\text { fines académicos. }\end{array}$ & $3,6 \%$ & $7,3 \%$ & $19,9 \%$ & $41,4 \%$ & $27,8 \%$ \\
\hline \multirow[t]{3}{*}{$\begin{array}{l}\text { Capacidad de } \\
\text { respuesta }\end{array}$} & $\begin{array}{l}\text { La Institución muestra interés en colaborar a los } \\
\text { estudiantes de forma rápida los servicios requeridos. }\end{array}$ & $4,1 \%$ & $10,7 \%$ & $31,7 \%$ & $40,7 \%$ & $12,8 \%$ \\
\hline & $\begin{array}{l}\text { El departamento de matrículas responde de manera } \\
\text { oportuna las solicitudes de los usuarios en los tiempos } \\
\text { establecidos para sus trámites académicos. }\end{array}$ & $5,8 \%$ & $14,0 \%$ & $29,1 \%$ & $39,5 \%$ & $11,6 \%$ \\
\hline & $\begin{array}{l}\text { El personal a cargo del departamento de matrículas, } \\
\text { ofrece alternativas de solución a los usuarios al } \\
\text { momento de presentarse una falla técnica que impida } \\
\text { realizar la gestión del proceso. }\end{array}$ & $4,1 \%$ & $15,7 \%$ & $31,5 \%$ & $40,4 \%$ & $8,2 \%$ \\
\hline \multirow[t]{2}{*}{$\begin{array}{l}\text { Infraestructura } \\
\text { física }\end{array}$} & $\begin{array}{l}\text { La Institución cuenta con capacidad de instalaciones } \\
\text { necesarias que les permita llevar a cabo de manera } \\
\text { eficiente todos los procesos de matrículas. }\end{array}$ & $3,1 \%$ & $12,1 \%$ & $21,3 \%$ & $47,9 \%$ & $15,5 \%$ \\
\hline & $\begin{array}{l}\text { Los equipos tecnológicos que tiene el departamento } \\
\text { de matrículas permiten que los trámites requeridos se } \\
\text { resuelvan eficazmente. }\end{array}$ & $3,9 \%$ & $7,5 \%$ & $28,8 \%$ & $46,0 \%$ & $13,8 \%$ \\
\hline \multirow[t]{4}{*}{$\begin{array}{l}\text { Interacción } \\
\text { humana }\end{array}$} & $\begin{array}{l}\text { La Institución se interesa por establecer una relación } \\
\text { constante con los usuarios, desde el momento en que } \\
\text { hacen parte de ella. }\end{array}$ & $3,1 \%$ & $13,6 \%$ & $28,8 \%$ & $40,7 \%$ & $13,8 \%$ \\
\hline & $\begin{array}{l}\text { Los colaboradores del departamento de matrículas } \\
\text { tienen en cuenta las sugerencias de los usuarios para } \\
\text { realizar mejoras en los procesos de servicio al cliente. }\end{array}$ & $5,8 \%$ & $14,5 \%$ & $38,3 \%$ & $32,2 \%$ & $9,2 \%$ \\
\hline & $\begin{array}{l}\text { Las observaciones y sugerencias por parte de los } \\
\text { usuarios, evidencian mejoras en los procesos de } \\
\text { matrículas de la Institución. }\end{array}$ & $4,6 \%$ & $11,4 \%$ & $36,8 \%$ & $37,0 \%$ & $10,2 \%$ \\
\hline & $\begin{array}{l}\text { El servicio otorgado por el departamento de } \\
\text { matrículas, resulta cordial y agradable para el usuario. }\end{array}$ & $3,1 \%$ & $7,3 \%$ & $21,8 \%$ & $46,5 \%$ & $21,3 \%$ \\
\hline
\end{tabular}


En las fases de análisis estadístico, una vez obtenido los datos se procedió a realizar el procesamiento de estos mediante dos pruebas de Fiabilidad (Alpha de Cronbach y Omega de McDonald). En términos métricos del instrumento, la primera prueba arrojó un estadístico de forma global que demuestra su consistencia interna en la medición de los ítems; el análisis por componentes de los valores obtenidos en capacidad de innovación con los factores organizacionales reafirma una vez más su confiabilidad y el Omega de McDonald presentó cargas factoriales con un estadístico que confirma la validez del instrumento aplicado, demostrando así unos resultados consistentes y afines con la situación planteada en el estudio ver tabla 4.

Tabla 4. Fiabilidad del instrumento

\begin{tabular}{|l|c|c|}
\hline & Alpha de Cronbach & Omega de McDonald \\
\hline Factores de Capacidad de Innovación & 0,867 & \multirow{2}{*}{0,96} \\
\hline Factores Organizacionales & 0,917 & \\
\hline Global & 0,940 & \\
\hline
\end{tabular}

La segunda prueba de análisis factorial (AF), se optó primero por un AF con estimaciones por componentes principales, rotación Varimax, para extraer lo que el modelo teórico explicaba, en este caso fueron 10 factores en total. A su vez, el determinante de la matriz de correlaciones fue de 7,5×10-7, la medida de adecuación muestral, arrojó un $\mathrm{KMO}=0.94$ con el que se compararon las magnitudes de los coeficientes de correlación parcial y la prueba de esfericidad de Barlett generó un Chi-cuadrado=5657,82 (435 grados de libertad) con un $p=0,000$, que indica que la matriz de correlación no era una matriz de identidad. Por tanto, los resultados afirman que el valor de cada indicador muestra la correlación de los datos y es aplicable para la realización del análisis factorial. Además, el modelo factorial reunió el 70,16\% de la varianza total y el valor más bajo de las comunalidades fue de 55,6\%. Es decir, que el modelo explicó la dispersión de las variables originales por encima del 55,6\% (llegando a explicar hasta el $80 \%$ de la variabilidad en algunos ítems), evidenciando que los resultados son confiables ya que explica en gran medida la totalidad los datos obtenidos. Ver la tabla 5.

Tabla 5. Dispersión de las variables originales

\begin{tabular}{|l|l|}
\hline Ítem & Extracción \\
\hline $\mathrm{p} 1$ & 0,700 \\
\hline $\mathrm{p} 2$ & 0,749 \\
\hline $\mathrm{p} 3$ & 0,716 \\
\hline $\mathrm{p} 4$ & 0,575 \\
\hline $\mathrm{p} 5$ & 0,689 \\
\hline p6 & 0,716 \\
\hline p7 & 0,633 \\
\hline p8 & 0,685 \\
\hline p9 & 0,737 \\
\hline p10 & 0,797 \\
\hline p11 & 0,658 \\
\hline p12 & 0,730 \\
\hline p13 & 0,768 \\
\hline p14 & 0,671 \\
\hline p15 & 0,701 \\
\hline
\end{tabular}

\begin{tabular}{|l|l|}
\hline Ítem & Extracción \\
\hline$p 16$ & 0,693 \\
\hline$p 17$ & 0,724 \\
\hline$p 18$ & 0,633 \\
\hline$p 19$ & 0,761 \\
\hline$p 20$ & 0,769 \\
\hline$p 21$ & 0,752 \\
\hline$p 22$ & 0,713 \\
\hline$p 23$ & 0,749 \\
\hline$p 24$ & 0,694 \\
\hline$p 25$ & 0,712 \\
\hline$p 26$ & 0,705 \\
\hline$p 27$ & 0,631 \\
\hline$p 28$ & 0,732 \\
\hline$p 29$ & 0,699 \\
\hline$p 30$ & 0,556 \\
\hline
\end{tabular}

La fase de análisis factorial confirmatorio, se verificó que las variables medidas estaban organizadas bajo la estructura factorial inicial, con una bondad de ajuste Chi-cuadrado=826.64 (360 grados de libertad), y $p=0,000$, indicando un buen ajuste de la muestra que se abordó en este estudio. A su vez, los índices de ajuste global fueron de 0.8819 y 0.8475 , el Error Medio Cuadrático RMSEA $=0.0560$ (IC: $0.051,0.061$ ), evidenciando un buen ajuste de las variables. En los índices de ajuste comparativo $\mathrm{CFI}=0.9131$, IFI=0.9143 y SRMR $=0.0460$, estos presentan un buen ajuste del modelo, también se observó relaciones de causalidad entre las variables latentes (factores organizacionales y capacidad de innovación) con los ítems originales, donde se confirma que todas las relaciones tienen un grado de estadística significativo al presentar un $p<0,05$, indican que la capacidad de innovación en IES, influye fuertemente en los procesos institucionales ocasionando un efecto positivo en los servicios que ofrecen las universidades a los estudiantes, además significa un factor clave en los procesos institucionales; ya que, su influencia con los factores organizacionales indica que la innovación puesta en práctica en IES contribuye al desarrollo de competencias desde el marco social del conocimiento, resaltando su capacidad de innovación y adaptación al cambio brindando servicios óptimos. Ver tablas 6 y 7. 
Tabla 6. Relaciones de causalidad entre las variables latentes con los ítems originales

\begin{tabular}{|c|c|c|c|c|c|c|c|}
\hline & Estimado & $\begin{array}{l}\text { Error } \\
\text { Estándar }\end{array}$ & Valor Z & $P$ & \multicolumn{3}{|c|}{ Causalidad } \\
\hline Iam[p1:Explotacion] & 0,71 & 0,05 & 15,31 & 0,000 & p1 & $<--$ & Explotación \\
\hline Iam[p2:Explotacion] & 0,76 & 0,05 & 16,90 & 0,000 & p2 & $<---$ & Explotación \\
\hline Iam[p3:Explotacion] & 0,75 & 0,05 & 16,54 & 0,000 & p3 & $<--$ & Explotación \\
\hline lam[p4:Exploracion] & 0,61 & 0,05 & 12,82 & 0,000 & p4 & $<--$ & Exploración \\
\hline lam[p5:Exploracion] & 0,57 & 0,05 & 11,88 & 0,000 & p5 & $<---$ & Exploración \\
\hline Iam[p6:Exploracion] & 0,64 & 0,05 & 13,39 & 0,000 & p6 & $<---$ & Exploración \\
\hline Iam[p7:Ambidestreza] & 0,71 & 0,04 & 15,95 & 0,000 & p7 & $<---$ & Ambidestreza \\
\hline Iam[p8:Ambidestreza] & 0,61 & 0,05 & 13,18 & 0,000 & p8 & $<---$ & Ambidestreza \\
\hline Iam[p9:Ambidestreza] & 0,70 & 0,04 & 15,62 & 0,000 & p9 & $<---$ & Ambidestreza \\
\hline lam[p10:OriCliente] & 0,69 & 0,05 & 14,21 & 0,000 & p10 & $<--$ & OriCliente \\
\hline Iam[p11:OriCliente] & 0,65 & 0,05 & 13,50 & 0,000 & p11 & $<---$ & OriCliente \\
\hline Iam[p12:OrialMercado] & 0,77 & 0,05 & 15,71 & 0,000 & p12 & $<---$ & OrialMercado \\
\hline Iam[p13:OrialMercado] & 0,77 & 0,05 & 15,86 & 0,000 & p13 & $<---$ & OrialMercado \\
\hline $\operatorname{lam}[\mathrm{p} 14:$ OrialaTecnol] & 0,67 & 0,05 & 14,53 & 0,000 & p14 & $<---$ & OrialaTecnol \\
\hline Iam[p15:OrialaTecnol] & 0,72 & 0,05 & 15,97 & 0,000 & p15 & $<---$ & OrialaTecnol \\
\hline Iam[p16:OrialaTecnol] & 0,78 & 0,04 & 17,90 & 0,000 & p16 & $<---$ & OrialaTecnol \\
\hline Iam[p17:OrialaTecnol] & 0,71 & 0,05 & 15,82 & 0,000 & p17 & $<---$ & OrialaTecnol \\
\hline Iam[p18:OrialaTecnol] & 0,54 & 0,05 & 11,17 & 0,000 & $\mathrm{p} 18$ & $<---$ & OrialaTecnol \\
\hline lam[p19:Fiabilidad] & 0,73 & 0,05 & 15,13 & 0,000 & $\mathrm{p} 19$ & $<---$ & Fiabilidad \\
\hline lam[p20:Fiabilidad] & 0,74 & 0,05 & 15,45 & 0,000 & p20 & $<---$ & Fiabilidad \\
\hline lam[p21:Fiabilidad] & 0,56 & 0,05 & 11,13 & 0,000 & p21 & $<--$ & Fiabilidad \\
\hline Iam[p22: Cap. de Respuesta] & 0,76 & 0,05 & 16,30 & 0,000 & p22 & $<--$ & Cap. de Respuesta \\
\hline Iam[p23: Cap. de Respuesta] & 0,67 & 0,05 & 13,80 & 0,000 & p23 & $<---$ & Cap. de Respuesta \\
\hline Iam[p24: Cap. de Respuesta] & 0,63 & 0,05 & 12,82 & 0,000 & p24 & $<---$ & Cap. de Respuesta \\
\hline lam[p25:Infraestructura] & 0,67 & 0,05 & 13,90 & 0,000 & $\mathrm{p} 25$ & $<---$ & Infraestructura \\
\hline lam[p26:Infraestructura] & 0,80 & 0,05 & 16,83 & 0,000 & p26 & $<--$ & Infraestructura \\
\hline $\operatorname{lam}[\mathrm{p} 27: \mathrm{IH}]$ & 0,66 & 0,05 & 14,26 & 0,000 & p27 & $<---$ & $\mathrm{IH}$ \\
\hline $\operatorname{lam}[\mathrm{p} 28: \mathrm{IH}]$ & 0,75 & 0,04 & 16,74 & 0,000 & $\mathrm{p} 28$ & $<---$ & $\mathrm{IH}$ \\
\hline Iam[p29:IH] & 0,73 & 0,04 & 16,36 & 0,000 & p29 & $<---$ & $\mathrm{IH}$ \\
\hline $\operatorname{lam}[\mathrm{p} 30: \mathrm{IH}]$ & 0,62 & 0,05 & 13,18 & 0,000 & p30 & $<<--$ & $\mathrm{IH}$ \\
\hline
\end{tabular}

Tabla 7. Significancia de las relaciones covariación entre los factores organizacionales y los de capacidad de innovación

\begin{tabular}{|c|c|c|c|c|c|c|c|}
\hline & Estimado & Error & Valor & $P$ & Causalidad & & \\
\hline C[Explotación, OriCliente] & 0,75 & 0,04 & 15,64 & 0,000 & OriCliente & $<-->$ & Explotación \\
\hline C[Explotación, OrialMercado] & 0,45 & 0,05 & 8,39 & 0,000 & OrialMercado & $<-->$ & Explotación \\
\hline C[Explotación, OrialaTecnol] & 0,64 & 0,04 & 15,63 & 0,000 & OrialaTecnol & $<-->$ & Explotación \\
\hline C[Explotación, Fiabilidad] & 0,42 & 0,05 & 7,57 & 0,000 & Fiabilidad & $<-->$ & Explotación \\
\hline C[Explotación, Cap. de Respuesta] & 0,55 & 0,05 & 10,94 & 0,000 & Cap. de Respuesta & $<-->$ & Explotación \\
\hline C[Explotación, Infraestructura] & 0,63 & 0,04 & 13,02 & 0,000 & Infraestructura & $<-->$ & Explotación \\
\hline $\mathrm{C}[$ Explotación, IH] & 0,69 & 0,04 & 17,43 & 0,000 & $\mathrm{IH}$ & $<-->$ & Explotación \\
\hline C[Exploración, OriCliente] & 0,96 & 0,04 & 19,64 & 0,000 & OriCliente & $<-->$ & Exploración \\
\hline C[Exploración, OrialMercado] & 0,64 & 0,05 & 12,04 & 0,000 & OrialMercado & $<-->$ & Exploración \\
\hline C[Exploración, OrialaTecnol] & 0,82 & 0,03 & 20,72 & 0,000 & OrialaTecnol & $<-->$ & Exploración \\
\hline C[Exploración, Fiabilidad] & 0,57 & 0,05 & 9,84 & 0,000 & Fiabilidad & $<-->$ & Exploración \\
\hline C[Exploración, Cap. de Respuesta] & 0,62 & 0,05 & 11,35 & 0,000 & Cap. de Respuesta & $<-->$ & Exploración \\
\hline C[Exploración, Infraestructura] & 0,81 & 0,04 & 17,07 & 0,000 & Infraestructura & $<-->$ & Exploración \\
\hline $\mathrm{C}[$ Exploración, IH] & 0,79 & 0,04 & 18,54 & 0,000 & $\mathrm{IH}$ & $<-->$ & Exploración \\
\hline C[Ambidestreza, OriCliente] & 1,06 & 0,04 & 26,16 & 0,000 & OriCliente & $<-->$ & Ambidestreza \\
\hline $\mathrm{C}[$ Ambidestreza, OrialMercado] & 0,57 & 0,05 & 10,84 & 0,000 & OrialMercado & $<-->$ & Ambidestreza \\
\hline C[Ambidestreza, OrialaTecnol] & 0,80 & 0,03 & 22,96 & 0,000 & OrialaTecnol & $<-->$ & Ambidestreza \\
\hline C[Ambidestreza, Fiabilidad] & 0,66 & 0,04 & 13,65 & 0,000 & Fiabilidad & $<-->$ & Ambidestreza \\
\hline C[Ambidestreza, Cap. de Respuesta] & 0,72 & 0,04 & 16,11 & 0,000 & Cap. de Respuesta & $<-->$ & Ambidestreza \\
\hline $\mathrm{C}[$ Ambidestreza, Infraestructura] & 0,80 & 0,04 & 18,89 & 0,000 & Infraestructura & $<-->$ & Ambidestreza \\
\hline $\mathrm{C}[$ Ambidestreza, $\mathrm{IH}]$ & 0,90 & 0,03 & 29,83 & 0,000 & $\mathrm{IH}$ & $<-->$ & Ambidestreza \\
\hline
\end{tabular}


En el estudio de las relaciones entre ambas variables, se optó por los coeficientes de correlación de Pearson, a partir de la matriz de correlaciones se afirma que las correlaciones entre los factores de servicio al cliente y capacidad de innovación fueron significativas al 1\% del nivel de significancia. Señalado una asociación lineal entre los factores de las variables estudiadas; a su vez, existen más correlaciones fuertes por encima de 0,5 entre los factores organizacionales de servicio al cliente con exploración y ambidestreza, y en menor grado con la explotación. Por ejemplo, al observar que la orientación al cliente, a la tecnología, interacción humana e infraestructura tienen al menos dos correlaciones por encima de 0,5 con los factores de capacidad de innovación; lo contrario, con fiabilidad, capacidad de respuesta y orientación al mercado manejan márgenes muy moderados, indicando la necesidad de mejoras en los procesos de servicio, ver tabla 8.

Tabla 8. Matriz de correlaciones ( ${ }^{* *}$ Significativa al 1\% bilateral.)

\begin{tabular}{|l|l|l|l|l|}
\hline & & \multicolumn{3}{|l|}{ Capacidad de Innovación } \\
\hline & & Explotación & Exploración & Ambidestreza \\
\hline \multirow{2}{*}{$\begin{array}{l}\text { Factores } \\
\text { organizacionales de } \\
\text { servicio al cliente }\end{array}$} & Orientación al cliente &, $538^{* *}$ &, $612^{* *}$ &, $703^{* *}$ \\
\cline { 2 - 5 } & Orientación al mercado &, $358^{* *}$ &, $452^{* *}$ &, $413^{* *}$ \\
\cline { 2 - 5 } & Orientación a la tecnología &, $534^{* *}$ &, $594^{* *}$ &, $609^{* *}$ \\
\cline { 2 - 5 } & Fiabilidad &, $321^{* *}$ &, $398^{* *}$ &, $472^{* *}$ \\
\cline { 2 - 5 } & Capacidad de respuesta &, $419^{* *}$ &, $433^{* *}$ &, $523^{* *}$ \\
\cline { 2 - 5 } & Infraestructura &, $475^{* *}$ &, $549^{* *}$ &, $561^{* *}$ \\
\cline { 2 - 5 } & Interacción humana &, $544^{* *}$ &, $563^{* *}$ &, $684^{* *}$ \\
\hline
\end{tabular}

\section{DISCUSIÓN}

Desde el enfoque teórico planteado sobre desarrollo de capacidad de innovación en procesos de servicio al cliente en el área de matrícula de las IES, se resalta lo planteado por Hernández et al., (2016) al indicar que la innovación es la base del cambio en el proceso o comportamiento de alguien al interior de la organización, para lograr un mejor desempeño en el mercado. Asimismo, Mohamad et al., (2010) afirman que las IES logran éxito en su desempeño innovador, si su desarrollo se orienta a capacidades organizacionales y al entorno en el que operan. Por su parte, Asma y Abdellatif (2016) en su estudio asevera que el empleo de estrategias innovadoras reconoce y garantiza conocimientos y aprendizajes generados en varios procesos de las IES que permiten aprovechar recursos y oportunidades para lograr el cumplimiento misional. No obstante, Benneworth et al., (2017) señala que la misión de las IES, se centra en la propiedad intelectual de los empleados, sobre los resultados de innovación en la educación superior, esto solo involucra actividades de I+D y la capacidad de innovación también implica habilidades emprendedoras del individuo e innovación en procesos de servicio al cliente. Asimismo, Reichert (2019) afirma que las IES son llamadas a interactuar, cocrear y alcanzar un nivel de impacto en el desarrollo local, nacional y global, que las denomine como IES de cuarta generación, que persiguen cambios institucionales de gran autonomía, aprendizaje, colaboración, interdisciplinariedad y aumento de servicios, con mayor apertura para interactuar con socios externos mediante innovación abierta. Finalmente, este estudio demuestra que las IES necesitan enfrentar los cambios del entorno, ofreciendo soluciones optimas a través de procesos innovadores que les permitan actuar rápida y eficientemente, esto es pertinente con lo expuesto por De Moortel y Crispeels (2018) al afirmar que el proceso de cambio basado en innovación permite a las IES diferenciación, mayor productividad y reconocimiento en el mercado.

\section{CONCLUSIONES}

De acuerdo a los resultados obtenidos en este estudio se determinó la importancia del desarrollo de capacidad de innovación en procesos de servicio al cliente y como favorece en la satisfacción del estudiante en relación con los trámites académicos que realizan en el departamento de matrícula de una IES. Por ende, la situación actual en las IES demuestra la necesidad de mejorar en esa área el desempeño del personal a cargo de los procesos académicos que se llevan a cabo allí, y puedan responder oportunamente al estudiante, superando sus expectativas con el servicio ofrecido y satisfacer plenamente sus necesidades. En este contexto, las IES como productoras de conocimiento, es importante que promuevan el desarrollo de habilidades innovadora en su equipo laboral, el cual debe estar alineado con los objetivos estratégicos institucionales. No obstante, las IES necesitan seguir fortaleciendo la explotación de sus recursos aprovechándolos para desarrollar y ofrecer servicios novedosos y excepcionales al cliente. Aunque las IES estudiadas presentan manejo tecnológico, sin embargo, la información en las plataformas casi siempre se muestra desactualizada, indicando mejoras en ese proceso, para que el estudiante pueda encontrar información clara, actualizada y oportuna. Finalmente, la innovación en servicios denota características muy particulares que indican la importancia de aprovechar los recursos y capacidades institucionales para crear servicios extraordinarios que evidencien la capacidad innovadora en el área de matrícula y su reconocimiento gracias a las buenas experiencias del estudiante. 


\section{AGRADECIMIENTOS}

Los autores agradecen el apoyo de la Universidad Simón Bolívar, de la Dirección de Investigación de la Universidad de La Serena-Chile y al Centro de Información Tecnológica de La Serena-Chile.

\section{REFERENCIAS}

Acosta, J. C., y Fischer, A. L., Condiciones de la gestión del conocimiento, capacidad de innovación y resultados empresariales. Un modelo explicativo. Pensamiento y Gestión, (35), 25-63, (2013).

Arias, J., Durango, C. M., y Millán, N. T., Capacidad de innovación de proceso y desempeño innovador: efecto mediador de la capacidad de innovación de producto. AD-minister, (27), 75-93, (2015).

Asma, K y M. Abdellatif., A New Model for the Impact of Knowledge Management on University Performance. Part 1: Theoretical Development, doi: 10.1142/S0219649216500416, Journal of Information y Knowledge Management, 15 (4) (2016).

Benneworth, P. S., Sánchez, M., y otros dos autores. Measuring the contribution of higher education to innovation capacity in the EU: Study. https://doi.org/10.2766/802127, Publications Office of the European Union. (2017).

Berry, L. L., Un buen servicio ya no basta: cuatro principios del servicio excepcional al cliente, 13-158. Grupo Editorial Norma, Bogotá-Colombia, (2013).

Botía, M. L., y Orozco, L. H., Competencias en servicio al cliente y efectividad en solución de problemas, Rev. Estudios y Perspectivas en Turismo, ISSN: 0327-5841, 21, (3), 646 - 662, (2012).

Chen, Y., y Yuan, Y., The Innovation Strategy of Firms: Empirical Evidence from the Chinese High-Tech Industry, doi: 10.1108/17468770710756095, Journal of Technology Management, 2, (2),145-153, (2007).

Da Silva, D. N., Vieira, R. K., y otros dos autores. Optimización del Proceso de Innovación para Proyectos Internos en las Empresas. https://dx.doi.org/10.4067/S0718-07642016000300011, Información tecnológica, 27, 3, 119-130, (2016).

De Moortel K. y Crispeels, T., International university-university technology transfer: Strategic management framework, doi: 10.1016/j.techfore.2018.05.002, Technological Forecasting and Social Change, 135, 145-155 (2018).

Duque, E. J., Revisión del concepto de calidad del servicio y sus modelos de medición, Rev. Innovar, ISSN: 0121-5051, 15, (25), $64-80$, (2005).

Espinosa, F. F., Días, A., y Back, N. Un Procedimiento de Evaluación de las Condiciones Necesarias para Innovar la Gestión de Mantenimiento en una Empresa. https://dx.doi.org/10.4067/S0718-07642008000100012, Información tecnológica, 19, 1, 97-104 (2008).

García, O., Quintero, J., y Arias, J., Capacidades de innovación, desempeño innovador y desempeño organizacional en empresas del sector servicios, Rev. Cuadernos de Administración, 27, (49), 87 - 108 (2014).

Gruca, T. S., y Rego, L. L., Customer satisfaction, cash $f l$ ow, and shareholder value, https://doi.org/10.1509/jmkg.69.3.1.66358, Journal of Marketing, 69, (3), 115-130, (2005).

Hayek, F. A., The use of knowledge in society. The American Economic Review, 35, (4), pp. 519 - 530, (1945).

Hernández, R., Fernández, C., y Baptista, P., Metodología de la investigación. 6ª edición, 6ta 1-634. Mc Graw Hill, México, (2014).

Hernández, J., Tirado, P., y Ariza, A., El concepto de innovación social: ámbitos, definiciones y alcances teóricos CIRIECEspaña, Rev. Economía Pública, Social y Cooperativa, ISSN: 0213-8093, 88, 164 - 199, (2016).

Hosseini, S. S., Nikkhah Tekmedash, Y. y otros dos autores, Y, The Impact of Knowledge Management Strategy on Service Innovation Performance in Private and Public Hospitals, doi: https://doi.org/10.22059/ijms.2018.249784.672966; Iranian Journal of Management Studies, 12(1), 1-24 (2019).

Jordán, J. C., La innovación: una revisión teórica desde la perspectiva de marketing. Perspectivas, ISSN: 19943733, (27), 47-71, (2011).

Kohler, H. D., y González, S., Elementos para un concepto sociológico de innovación, https://doi.org/10.5944/empiria.29.2014.12942, Metodología de Ciencias Sociales, 29, 67 - 88, (2014).

Lloret, S., Ferreres, A., y otros dos autores., El análisis factorial exploratorio de los ítems: una guía práctica, revisada y actualizada, doi: 10.6018/analesps.30.3.199361, Anales de Psicología, 30, (3), 1151-1169, (2014).

Michelino, F., E. Lamberti y otros dos autores, Open models for innovation: an accounting-based perspective, https://doi.org/10.1504/IJTM.2015.068778, International Journal of Technology Management, 68(1-2), 99-121 (2015).

Mohamad, K., Ahmad, T., y Radiah. O. "Innovation capacity in higher education," https://doi.org/10.1109/cssr.2010.5773738, International Conference on Science and Social Research, Kuala Lumpur, Malaysia, pp. 1295-1300, (2010). 
Narver, J.C., Slater, S.F. y MacLachlan, D.L., Responsive and proactive market orientation and newproduct success, https://doi.org/10.1111/j.0737-6782.2004.00086.x, Journal of Product Innovation Management, 21, (5), 334-347, (2004).

OCDE, \& Eurostat. Oslo Manual: Guidelines for Collecting and Interpreting Innovation Data (Third ed.). Oslo: OCDE Eurostat, (2006).

Reichert, S., The Role of Universities in Regional Innovation Ecosystems. EUA Study. European University Association, (2019).

Schumpeter, J. A., Capitalismo, Socialismo y Democracia. Página Indómita, Madrid-España, (1942).

Sözbilir, F. Innovation Capacity and Innovation Performance in Terms of Educational Level of Managers. İşletme Araştırmaları Dergisi, 10, 2, 1-12 (2018).

Yam, R. C., Guan, J. C., y otros dos autores., An audit of tecnological innovation capabilities in Chinese firms: some empirical findings in Beijing, doi: 10.1016/j.respol.2004.05.004, Research policy,33, (8), 1123 - 1140, (2004).

Valbuena, R. E., La Investigación Científica Avanzada: Con Introducción a los Programas de Investigación Científica, La Investigación Internivel y el Razonamiento Artificial. Zulia-Venezuela (2017). 